\title{
Experimental Measurements of Permeability of Open Foam
}

\author{
Muath Alomair, Syeda Humaira Tasnim \\ School of engineering, University of Guelph \\ 50 Stone Road East, Guelph, ON Canada
}

\begin{abstract}
Open cell metal and carbon foams have unique properties that make them very attractive to a wide range of engineering applications such as heat transfer in heat exchanger analysis and design, petroleum engineering, aerospace engineering, chemical engineering, fuel cell technology, filtration, and flow stabilization. The study of heat and fluid flow through such foams or porous media depends on a series of unique concepts such as porosity, permeability, and volume averaged pressure drop and it is very essential that these concepts should be properly understood. This paper reports the experimental measurements of the porosity and permeability of open cell aluminum and reticulated vitreous carbon (RVC) foams of pore sizes varying from10 ppi (pores per inch) to 100 ppi. An experimental setup is developed and measurements are taken.
\end{abstract}

Keywords: Aluminum Foam, Reticulated Vitreous Carbon Foam, Porosity, Permeability, Experimental Measurements.

\section{Introduction}

The porous medium finds its application in various fields such as in petroleum engineering, chemical engineering, fuel cell technology, and heat transfer engineering. Porous materials are those which have voids to allow the fluid to pass through it. These porous media have benefit of enhanced heat exchange rate, due to large surface area, and ultimately decreasing the size of the whole product [1]. To know about the transportation aspect of the porous media, the porosity and permeability of the porous media should be determined, where the permeability is the ability of a porous medium to grant access to flow of fluid through it. These criteria of porous media need to be understood for their application in fuel cells where a gas diffusion layer is needed for the supply of fuel gas in a PEM fuel cell. These physical and thermal properties are governing factors for the efficiency of the PEM fuel cells. Even for the filtration application of porous media, the porosity and permeability play the major part [2]. In the Resin Transfer Moulding technique, much accurate value of permeability is needed to predict and simulate the flow behaviour of resin in the matrix to prevent any voids or defects [3]. Evaluation of porosity and permeability of porous media plays a vital role in analyzing heat transfer problems in engineering. Simulations of heat transfer problems in porous medium require the determination of the porosity and permeability constant of the desired materials [1] and [3].

\subsection{Review of Theory}

Darcy's law [4] is used to calculate permeability if the Reynolds number $(\mathrm{Re})$ of the flow is in between 1-10. The Re in this study is more than 10 which restricts the use of Darcy's relation. Thus, the modified Darcy equation, that is, DarcyForchheimer equation according to Bejan [4] is used to calculate the permeability. Darcy-Forchheimer equation [4] takes into account the inertia force term and is given below as

$$
-\frac{d P}{d y}=\frac{\mu}{\mathrm{K}} u+b \rho u^{2}
$$

Where $\mathrm{P}$ is the pressure, $\mathrm{y}$ is the vertical distance, $\mathrm{u}$ is the velocity, $\rho$ is the density, $\mu$ is the viscosity, $\mathrm{K}$ is the permeability, and $\mathrm{b}$ is the inertia coefficient, respectively.

A number of experimental analyses to determine the permeability of different porous media using different methods have been successfully carried out by using testing fluid such as water, air, and different gases. The values of permeability obtained experimentally for porous samples vary slightly depending on the testing fluid used and ambient temperature [5]. Miguel et al. [5] performed an experimental study to determine the permeability of a woven fabric using air and helium gas 
as testing fluid. They suggested that the flow of fluid through the void matrices of a porous material is propelled by the pressure difference and free molecular flow and the effective permeability which is a combined consequence of both its intrinsic permeability and its slip-flow permeability [5]. Vernet [6] also developed experimental setup and procedures to obtain the permeability of the carbon fabric. A comprehensive experimental work was conducted by Paek et al. [1] to study the effective conductivity and permeability of aluminum foam. The authors investigated the relevant dynamic features of the metal foam that influence its permeability and effective thermal conductivity. Their results showed that the permeability of the aluminum foam is typically affected by its cell size and porosity. Their experimental analysis also suggested the features a metal foam should possess for an optimal amount of heat transfer and less pressure drop for heat transfer application systems. Regulski et al. [7] conducted an experimental study to test the morphology and pressure drop of ceramic foams. The authors compared the experimental results with their numerical simulation results and other data existing in the literature.

The permeability of porous materials used in aerospace applications change during flight due to a chemical phenomenon known as Coke formation. Nicolas Gascoin et al. [8] studied the permeability constant of a porous material used in aerospace applications by using an infra-red signal modifications of a tracer injected through the porous material. The authors showed that the Darcian permeability can be measured during flight by analyzing the infra-red signals and linking them to their respective Darcian permeability. Gailani et al. [9] determined the permeability and porosity of human bone by using the data obtained experimentally from the stress-relaxation tests.

Other indirect methods of measuring the permeability of porous materials are illustrated by the use of reflected acoustic wave which was recently developed for porous materials of low resistivity, in which the use of a calibrated microphone was employed to obtain frequency values of the acoustic waves reflected from the porous media [10]-[11]. Experimental investigations were carried out to measure the permeability and porosity of a porous vertebral bone [12] and the results demonstrated that the intrinsic bone permeability depends on the bone porosity.

The current study measures the porosity and permeability of open cell metal and carbon foams. Aluminum foam samples of pore sizes 10, 20, and $40 \mathrm{ppi}$, RVC samples of pore sizes 10, 20, 30, 45, 80, and 100 ppi are considered. An experimental setup is developed for the steady flow of water as the working fluid.

\section{Experimental Setup}

An experimental steup is constructed in order to calculate the permeability of the porous samples experimentally in the lab environment. It consists of a $75 \mathrm{~L}$ tank, valve, differential pressure transducer (Benetech GM510), collecting beaker, and a timer as shown in Fig. 1(a) and (b). The porous samples are purchased from Duocel®.

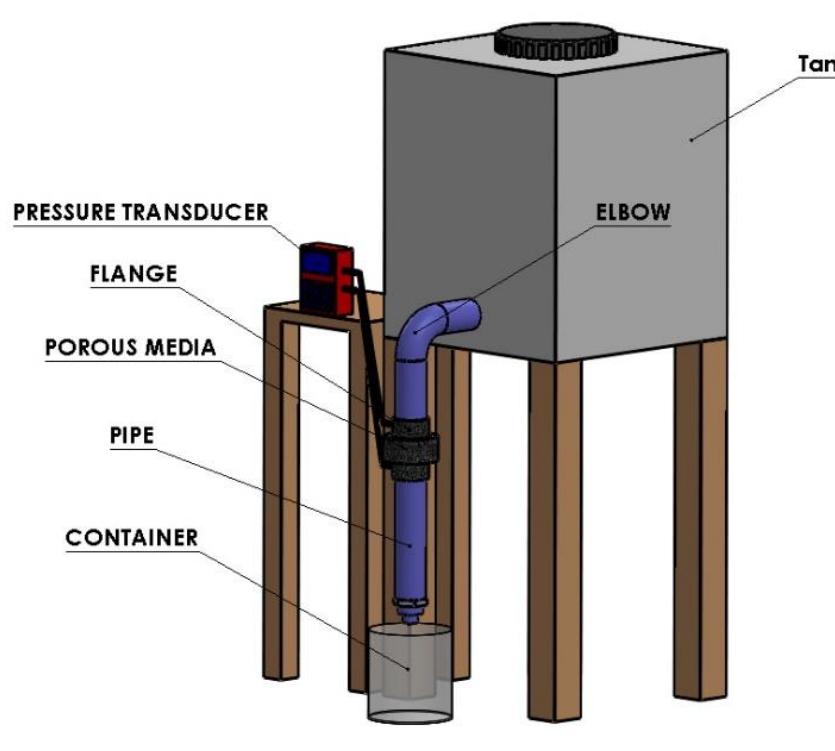

(a)

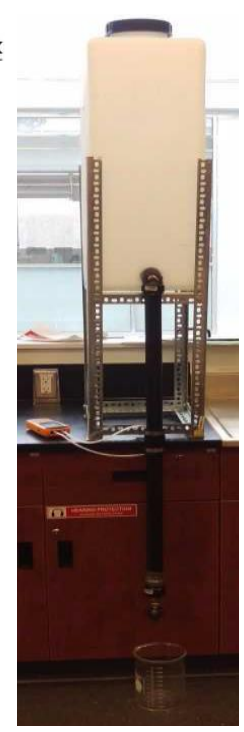

(b)

Fig. 1: (a) 3D image of the setup; (b) Experimental setup. 
Initially, three samples of aluminum foams with 10ppi, 20 ppi and 40 ppi are taken of dimensions $88.9 \times 38.1 \times 12.7 \mathrm{~mm}$ each. Six samples of RVC foam of $10 \mathrm{ppi}, 20 \mathrm{ppi}, 30 \mathrm{ppi}, 45 \mathrm{ppi}, 80 \mathrm{ppi}$ and $100 \mathrm{ppi}$ are taken having same dimensions for length and width as $88.9 \times 38.1 \mathrm{~mm}$ but the thickness of each sample varies as $6.35 \mathrm{~mm}, 6.86 \mathrm{~mm}, 6.35 \mathrm{~mm}, 5.33 \mathrm{~mm}, 6.86$ $\mathrm{mm}$ and $6.35 \mathrm{~mm}$ respectively. After measuring the porosity of the samples, these are cut into circular cross-section of diameter $34 \mathrm{~mm}$ each. The samples after machining process are shown in Fig. 2. The samples are cut $1 \mathrm{~mm}$ less than the diameter of the holder to seal the sample with Teflon tape to prevent any seepage of fluid through the wall of the samples.

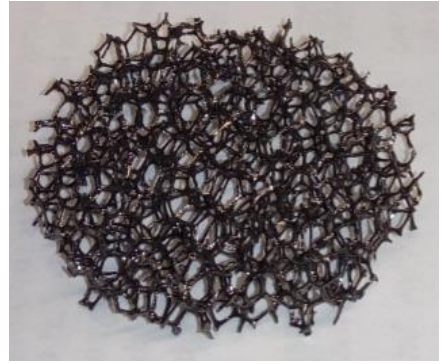

(a) 10 ppi

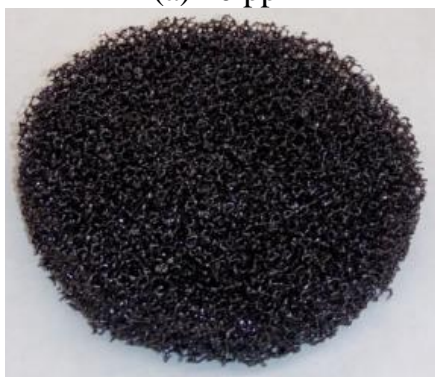

(d) 45 ppi

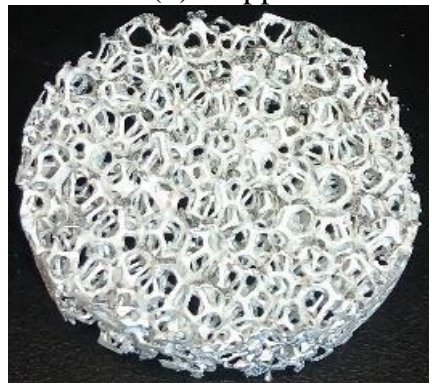

(g) $10 \mathrm{ppi}$

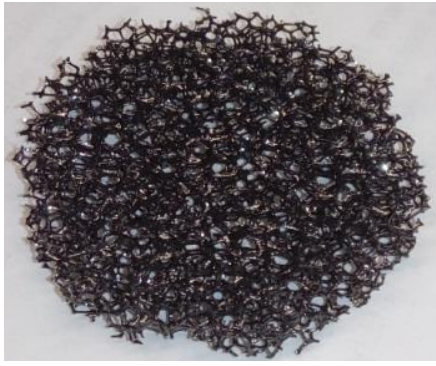

(b) 20 ppi

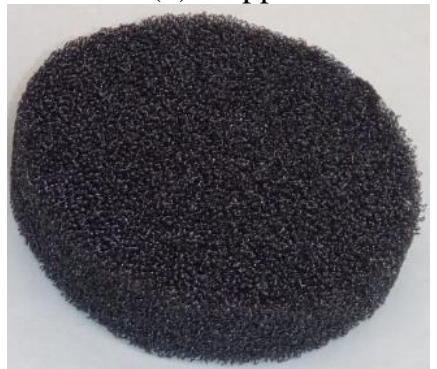

(e) 80 ppi

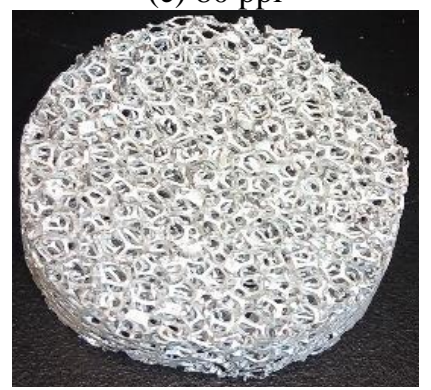

(h) $20 \mathrm{ppi}$

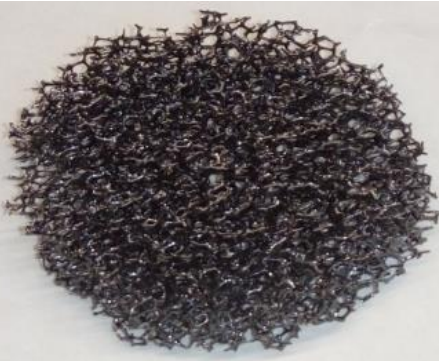

(c) $30 \mathrm{ppi}$

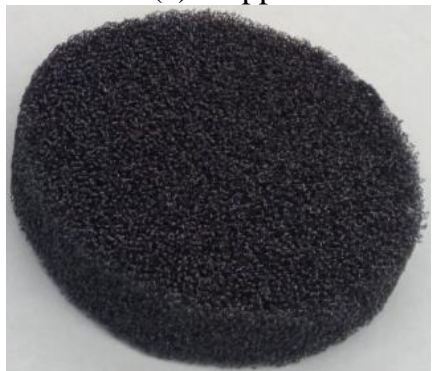

(f) $100 \mathrm{ppi}$

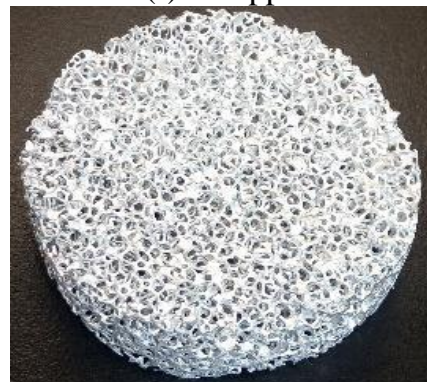

(i) $40 \mathrm{ppi}$

Fig. 2: Porous media samples: (a) to (f) carbon foam samples and (g) to (i) aluminum foam samples.

The porosities of samples are measured using the $400-\mathrm{mL}$ beaker. The initial reading of water in the beaker is noted down. Then, the uncut sample is immersed in the beaker and the rise in level of water is measured. The change in the level of water $(\Delta h)$ gives the volume of water displaced by the solid matrix of the porous medium, which is equivalent to the volume of the solid matrix. It can be calculated as:

$$
\begin{gathered}
V_{\text {solid }}=\pi R^{2} \Delta h \\
V_{\text {void }}=V_{\text {total }}-V_{\text {solid }}=l w t-\pi R^{2} \Delta h
\end{gathered}
$$

where 1 and $\mathrm{w}$ is the length and width of sample, $\mathrm{t}$ is thickness of the porous sample, $\mathrm{R}$ is the radius of measuring beaker and $\Delta h$ is rise in water level. 
The permeability is measured for the given samples using the apparatus as shown in Fig. 1. The tank at the top is filled with water and the water flows through the connected pipes, with the effect of gravity. No pump is incorporated as is used in other literatures [13]. The water flows in the pipes and through the porous medium, and is collected in the beaker at bottom. The pressure transducer is connected to two holes of $3 \mathrm{~mm}$ diameter, being $100 \mathrm{~mm}$ apart. The inlet and outlet units of the pressure transducer are attached across the coupling (porous sample) as shown in Fig. 1. The tank is filled with water up to 5-gallon level, and the water is released using the valve to allow the flow pass through the sample. The pressure across the sample changes and is recorded using the pressure transducer. The discharge of water is calculated by measuring water flowing out in a given time period (30 seconds). For this experimental setup, maintaining a very low flow rate by reducing the speed of the water flowing through the porous samples gave a negligible pressure difference reading, so the water flow rate was increased to obtain a measurable pressure difference value, which consequently resulted in Reynolds number values above 10 which rendered Darcy's law invalid for this experimental setup. Hence, we adopted Darcy's modified flow model (Darcy- Forchheimer model) for calculating the permeability. Furthermore, the gravitational force term (weight per unit volume) was also incorporated since the flow was induced by gravity [13] and [14]. Along with this modification, the modified Darcy-Forchheimer's equation is given below:

$$
K=\frac{\mu u+b \rho u^{2}}{-\frac{d P}{d y}+\rho g}
$$

Where $\mathrm{g}$ is the gravitational acceleration.

For the measurement of permeability of other samples, the water tank is emptied to remove the current sample and replace it with new one. Same procedure is followed, as discussed above, to record the different values of pressure gradient and the flow velocity to calculate the permeability values.

\section{Results and Discussions}

The main difficulty seen with the porosity measured was the presence of air bubbles while immersing the samples of high ppi values (80 - 100 PPI) because of very small pore diameter. It was resolved by using running water to pre-fill the pores of samples to some extent reducing the error in measurement. The porosity values were ranging from 0.5415 to 0.9771 for the given porous media samples. The measured values of the porosity of different samples are clearly increasing with the increase in the pores per linear inch. The minimum porosity was observed for the RVC foam sample of 100 ppi and the maximum was noted for RVC foam sample with 10 ppi because of the variation in pore size. For the measurement of permeability, the other parameters such as viscosity and density of water were taken as $\mu=1.002 \times 10-3 N s / \mathrm{m}^{2}$, and the density of water, $\rho=1000 \mathrm{~kg} / \mathrm{m}^{3}$ at $20^{\circ} \mathrm{C}$. Table 1 and 2 provide the values of porosity and permeability of different samples.

Table 1: The porosity and permeability values of aluminum foams.

\begin{tabular}{|c|c|c|}
\hline \multicolumn{2}{|c|}{ Aluminum samples } \\
\hline PPI & Porosity & Permeability $\left(\mathrm{m}^{2}\right)$ \\
\hline 10 & 0.9208 & $24.38 \times 10^{-8}$ \\
\hline 20 & 0.8812 & $19.42 \times 10^{-8}$ \\
\hline 40 & 0.7756 & $9.40 \times 10^{-8}$ \\
\hline
\end{tabular}


Table 2: The porosity and permeability values of RVC foams.

\begin{tabular}{|c|c|c|}
\hline \multicolumn{3}{|c|}{ RVC samples } \\
\hline PPI & Porosity & Permeability $\left(\mathrm{m}^{2}\right)$ \\
\hline 10 & 0.9771 & $56.12 \times 10^{-8}$ \\
\hline 20 & 0.9607 & $48.65 \times 10^{-8}$ \\
\hline 30 & 0.8680 & $41.72 \times 10^{-8}$ \\
\hline 45 & 0.7894 & $35.11 \times 10^{-8}$ \\
\hline 80 & 0.7151 & $18.48 \times 10^{-8}$ \\
\hline 100 & 0.5415 & $7.99 \times 10^{-8}$ \\
\hline
\end{tabular}

\section{Conclusion}

In this work, porosity and permeability of distinct porous samples were obtained using the experimental setup created. The Darcy Forchheimer equation along with gravity force term is used to calculate the permeability of different porous samples. During the measurement, it was noted that the reproduction of the permeability values for a certain sample is difficult. Some errors persist while measuring the permeability for the same sample repetitively. These errors include human errors, measurement error, and error with the pressure measuring device. The uncertainties in porosity is due to some air bubbles trapped in the porous medium and measurement error and is estimated to be $\pm 0.02 \%$. The pressure transducer has an accuracy of $\pm 0.3 \%$ at $20^{\circ} \mathrm{C}$ and repeatability error of $\pm 0.2 \%$. The results regarding the permeability also depend upon the sealing of the porous media samples. No or improper sealing of the samples lead to major alterations in the results obtained due to bypassing or escaping of fluid from the sides/periphery of the sample, as observed during the measurements. The ultimate objective of our work is to develop correlations for pressure drop based on geometrical characteristics of open cell foams.

\section{Acknowledgements}

The second author would like to express her sincere gratitude to graduate students Robin Singh and Ezike Kelechukwu for their support in preparing the experimental setup.

\section{References}

[1] J. W. Paek, et al, "Effective thermal conductivity and permeability of aluminum foam materials," International Journal of Thermophysics, vol. 21, no. 2, pp. 453-464, 2000.

[2] A. Tamayol, and M. Bahrami, "In-plane gas permeability of proton exchange membrane fuel cell gas diffusion layers," Journal of Power Sources, vol. 196, no. 7, pp. 3559-3564, 2011.

[3] K. Hoes, et al, "New set-up for measurement of permeability properties of fibrous reinforcements for RTM," Composites Part A: applied science and manufacturing, vol. 33, no. 7, pp. 959-969, 2002.

[4] A. Bejan, Convection Heat Transfer, 3rd ed. John Wiley \& Sons, INC., 2004.

[5] A. F. Miguel, and A. Serrenho, "On the experimental evaluation of permeability in porous media using a gas flow method," Journal of Physics D: Applied Physics, vol. 40, no. 21, p. 6824, 2007.

[6] N. Vernet, et al, "Experimental determination of the permeability of engineering textiles: Benchmark II," Composites Part A: Applied Science and Manufacturing, vol. 61, pp. 172-184, 2014.

[7] W. Regulski, et al. "Pressure drop in flow across ceramic foams-A numerical and experimental study," Chemical Engineering Science, vol. 137, pp. 320-337, 2015.

[8] N. Gascoin, F. Guillaume, and P. Gillard, "Determination of Darcian permeability of porous material by infrared spectrometry," Journal of Porous Materials, vol. 19, no. 3, pp. 317-331, 2012.

[9] G. Gailani, et al, "Experimental determination of the permeability in the lacunar-canalicular porosity of bone," Journal of biomechanical engineering, vol. 131, no. 10, p. 101007, 2009.

[10] A. Berbiche, et al, "Experimental determination of the viscous flow permeability of porous materials by measuring reflected low frequency acoustic waves," Journal of Applied Physics, vol. 119, no. 1, p. 014906, 2016.

[11] L. Luquot, and P. Gouze, "Experimental determination of porosity and permeability changes induced by injection 
of CO2 into carbonate rocks," Chemical Geology, vol. 265, no. 1-2, pp. 148-159, 2009.

[12] G. Baroud, et al, "Experimental and theoretical investigation of directional permeability of human vertebral cancellous bone for cement infiltration," Journal of biomechanics, vol. 37, no. 2, pp. 189-196, 2004.

[13] Z. Lafhaj, G. Richard, M. Kaczmarek, and F. Skoczylas, "Experimental determination of intrinsic permeability of limestone and concrete: Comparison between in situ and laboratory results," Build. Environ., vol. 42, no. 8, pp. 3042-3050, 2007.

[14] D. Poulikakos and A. Bejan, "Natural convection in a porous layer heated and cooled along one vertical side," Int. J. Heat Mass Transf., vol. 27, no. 10, pp. 1879-1891, 1984. 